\title{
Community-acquired pneumonia
}

\author{
Jeremy S Brown
}

\begin{abstract}
Community-acquired pneumonia (CAP) is one of the most common acute infections requiring admission to hospital. The main causative pathogens of CAP are Streptococcus pneumoniae, influenza A, Mycoplasma pneumoniae and Chlamydophila pneumoniae, and the dominant risk factors are age, smoking and comorbidities. The incidence of CAP and its common complications, such as the requirement for intensive care and complicated parapneumonic effusions, are increasing, making it essential for all physicians to have a good understanding of the management of CAP. Although the diagnosis and treatment of CAP is straightforward in most cases, it can be more complex, and recent data indicate that the mortality of CAP in the UK is surprisingly high. In the future, routine use of biomarkers to improve risk stratification and tailor management to individual patients could improve outcomes, and there is some evidence that modulation of CAP-associated inflammation could also be beneficial. Both research into host-microbial interactions in the lung and clinical trials of different management and preventative treatments are urgently needed to combat the increasing morbidity and mortality associated with CAP.
\end{abstract}

KEY WORDS: community-acquired pneumonia, infectious disease, biomarkers

\section{Introduction}

Community-acquired pneumonia (CAP) is one of the most common serious infective diseases, accounting for nearly $1 \%$ of all medical admissions. ${ }^{1}$ The incidence of CAP in the UK increased by 34\% between 1997 and 2005, ${ }^{1}$ and the rates of serious complications of CAP, such as admissions to intensive care and complicated parapneumonic effusions (CPE), are also on the increase. ${ }^{2,3}$ This growing burden of disease means that a good understanding of the management of CAP is necessary for any physician caring for acute admissions. Although the diagnosis and management of CAP should be straightforward in most cases, it can be more complex, and recent data indicate that the mortality of CAP in the UK is surprisingly high. ${ }^{4}$ Here, I provide some background on CAP and address some of the areas of difficulty in managing patients with this disease.

Jeremy S Brown, 1,2 professor of respiratory infection and honorary consultant in respiratory medicine

${ }^{1}$ University College London; ${ }^{2}$ University College London Hospitals Trust

\section{Causative agents of CAP}

The bacterial aetiology of CAP is well established and dictates the choice of empirical antibiotic therapy. The most common causative agent is Streptococcus pneumoniae, which is responsible for almost $50 \%$ of cases (Table 1) $;{ }^{5}$ other common causes are respiratory viruses (mainly influenza $\mathrm{A}$ ) and the atypical bacteria Chlamydophila pneumoniae and Mycoplasma pneumoniae. Less common bacterial causes are Haemophilus influenzae, Staphylococcus aureus, Moraxella catarrhalis and Legionella pneumophila. A significant number of patients with CAP have no microbial cause identified, even after extensive testing; ${ }^{5}$ whether these cases are the result of a novel pathogen or of false negative testsforestablished pathogensremains unknown. Microorganisms causing CAP reach the lungs either by inhalation of droplets created by sneezing or coughing from an infected contact (eg respiratory viruses, C. pneumophila and M. pneumoniae) or environmental source (L. pneumophila), or by microaspiration after colonisation of the nasopharynx with a potential pathogen (eg $S$. pneumoniae, $H$. influenzae or S. aureus). The reasons for the dominance of $S$. pneumoniae as a cause of CAP compared with other common nasopharyngeal commensals are poorly understood, but if elucidated could help identify novel preventative strategies.

\section{Immunology and risk factors}

Microbial exposure to the lung is constant, but the normal lung has effective immune mechanisms that usually prevent pneumonia. A comprehensive integrated understanding of the normal mechanisms of lung immunity is lacking, but many of the individual components are well known. Initial immune defence depends on effective mucociliary clearance and an intact epithelial barrier, supported by antimicrobial proteins or peptides, such as lactoferrin, lysozyme and defensins. In addition, the airways and alveoli contain alveolar macrophages that recognise and phagocytose invading microorganisms via a range of surface proteins, including scavenger receptors, macrophage receptor with collagenous structure (MARCO), dectin, complement and mannose receptors assisted by opsonins (complement, antibody and surfactants) in the airway-lining fluid. Failure to control invading microorganisms by these initial immune mechanisms triggers an inflammatory response, causing an influx of exudate and white cells into the alveoli and resulting in consolidation, the hallmark of pneumonia. Subsequently, bacteria can potentially invade the blood, where they can be controlled by complement and antibody in combination with phagocytosis by macrophages of the reticuloendothelial system and circulating neutrophils. Additional research is required to identify the relative importance 
Table 1. Sources of infection and clinical presentation of deep lung infections resulting from important community-acquired microbial

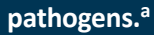

\begin{tabular}{|c|c|c|}
\hline Mode of lung invasion & Pathogen & Clinical presentation \\
\hline \multirow[t]{5}{*}{ Inhalation of infected droplets } & Influenza A & CAP (19\%) and/or bronchiolitis ${ }^{b}$ \\
\hline & Other respiratory viruses & CAP (6.1\%) and/or bronchiolitis \\
\hline & Chlamydophila pneumoniae & CAP (13\%) and/or bronchiolitis \\
\hline & Mycoplasma pneumoniae & CAP $(3+\%)$ and/or bronchiolitis \\
\hline & Mycobacterium tuberculosis & Subacute lung infection ${ }^{c}$ \\
\hline \multirow{7}{*}{$\begin{array}{l}\text { Aspiration of oral and/or } \\
\text { nasopharyngeal commensal }\end{array}$} & Streptococcus pneumoniae & CAP $(48 \%)$ \\
\hline & Haemophilus influenzae & CAP (7\%) \\
\hline & Staphylococcus aureus & CAP $(1.5 \%)$ \\
\hline & Moraxella catarrhalis & CAP $(2 \%)$ \\
\hline & Gram-negative enteric bacteria & CAP (1.4\%) \\
\hline & & Subacute lung infection ${ }^{c}$ \\
\hline & Actinomyces species & Subacute lung infection ${ }^{c}$ \\
\hline \multirow[t]{6}{*}{ Environmental source } & Legionella pneumophila & CAP (3\%) \\
\hline & Pseudomonas aeruginosa & $\operatorname{CAP}(<1 \%)$ \\
\hline & & Subacute lung infection ${ }^{c}$ \\
\hline & Nocardia species & Subacute lung infection ${ }^{c}$ \\
\hline & Aspergillus species & Subacute lung infection ${ }^{c}$ \\
\hline & Non-tuberculous mycobacteria & Subacute lung infection ${ }^{c}$ \\
\hline \multirow[t]{2}{*}{ Metastatic spread via septicaemia } & Staphylococcus aureus & Multiple nodules and abscesses ${ }^{d}$ \\
\hline & Fusobacterium necrophorum & Multiple nodules and abscesses ${ }^{d}$ \\
\hline \multicolumn{3}{|c|}{ aThe proportion of CAP cases associated with a particular pathogen is shown in brackets (source, Lim et al. 2001)..$^{5}$} \\
\hline \multicolumn{3}{|c|}{$\begin{array}{l}\text { 'Widespread infection of the small airways associated with distinctive 'tree-in-bud' appearances on CT scan and less obvious bilateral interstitial changes on chest radiograph. } \\
\text { 'Alveolar infection, usually presenting with a longer history ( }>3 \text { weeks), less hypoxia and often a weaker inflammatory response compared with patients with CAP. Chest } \\
\text { radiograph changes include consolidation (frequently non-lobar in distribution) and nodules; cavitation is common. } \\
\text { dClinical presentation can be similar to CAP but the radiology demonstrates bilateral multiple nodules, usually in a peripheral distribution, which often cavitate and erode into } \\
\text { the pleural space to cause hydro- and/or pyopneumothoraces. Staphylococcus aureus septicaemia can be caused by an infected venous catheter or right-sided endocarditis; } \\
\text { Fusobacterium necrophorum infections occur as a local spread from the pharynx to cause septic thrombophlebitis of the jugular vein (Lemierre's syndrome). }\end{array}$} \\
\hline
\end{tabular}

of each of these components for preventing CAP and the potential roles of $\mathrm{T}$ cell and other lymphocyte subsets which experimental data suggest are important.

Risk factors for CAP are largely defined by conditions that reduce the efficacy of the normal mechanisms of lung immunity (Table 2). The dominant risk factor is age, with the incidence of CAP increasing markedly in patients who are over 65 years of age (Table 2). As a consequence, CAP affects $2 \%$ of people over 85 years of age each year ${ }^{1}$ and, in a recent British Thoracic Society (BTS) UK-wide audit of CAP, the mean age of patients was 76 years. ${ }^{4}$ Why increasing age is closely related to the incidence of CAP is not clear, but will be multifactorial. Increasing age is associated with an increasing incidence of coexisting disease that could predispose to CAP; for example, chronic lung disease will reduce the efficiency of mucociliary clearance and neurological comorbidities might increase aspiration, adding to the bacterial load reaching the lungs. Aging increases the expression of the mucosal cell surface proteins that bacteria can adhere to, enabling potential pathogens to better avoid normal clearance mechanisms. ${ }^{6}$ There is also evidence for direct effects of age on aspects of innate and adaptive immunity, a process called immunosenescence, which will weaken lung immunity to invading microbes. ${ }^{7}$

\section{CAP diagnosis and disease severity}

Diagnosis of CAP is frequently obvious, with most patients presenting with the combination of evidence for acute infection and new consolidation, usually in an asymmetric lobar distribution. Consolidation is visible on the chest radiograph in over $90 \%$ of patients, but occasionally lags behind the clinical presentation and can be difficult to discern in the left lower lobe because of the heart shadow. CAP resulting from $M$. pneumoniae and $C$. pneumophila can present as a predominately small airways infection (a bronchiolitis) rather than as lobar consolidation, causing interstitial changes that are easily missed on poor-quality chest radiographs but readily identified on a CT scan as 'tree-in-bud' changes.

Patients with CAP should be assessed for disease severity using the CURB-65 score (or its derivative CRB65). ${ }^{8}$ The CURB-65 score dictates optimum empirical antibiotic therapy, and whether the patient can be treated safely at home (scores of 0 or 1 ) or 
Table 2. Defects in lung immunity that result in CAP and their associated risk factors.

Immune mechanism

Increased risk of microaspiration

Impaired mucociliary clearance and/or epithelial integrity

Impaired phagocyte function (alveolar macrophages and neutrophils)

Impaired adaptive immunity

\section{Risk factor for CAP}

Age?

Neurological disease

Drug or alcohol abuse

Smoking

Respiratory viral infections

Pre-existing lung disease

Air pollution?

Age?

Influenza (other viruses?)

Smoking?

Alcohol abuse and/or cirrhosis

Exposure to welding fumes?

Systemic corticosteroids

Inhaled corticosteroids?

Inherited and/or acquired complement deficiencies

Inherited and/or acquired antibody deficiencies

Age?

Inherited and/or acquired antibody deficiencies

Role for T cells?

Age?

HIV infection (with normal CD4 count)

Previous CAP

\section{Notes}

Incidence/1000: <65 yr, 0.8; 65-74 yr, 3.5; 75-84 yr, 8.8; $>85$ years, 22

Attributable risk is $32 \%$

eg increased expression of microbial ligands for adhesion (eg platelet-activating factor receptor)

Reduced recognition of invading bacteria and increased inflammatory responses

eg in chronic obstructive pulmonary disease

eg C2 deficiency, systemic lupus erythematosus; high incidence of Streptococcus pneumoniae infection

eg common variable immunodeficiency and multiple myeloma

Immunosenescence?

eg common variable immunodeficiency and multiple myeloma

Supported by experimental data

Immunosenescence?

Very high risk with odds ratio (OR) of 18

Presumably because of the persistence of the above risk factor(s)

$\mathrm{CAP}=$ community-acquired pneumonia

should be admitted to a general (a score of 2) or possibly an intensive care ward (a score of 3+). Although it has been extensively validated and is simple to use, the CURB-65 score has limitations. It is poor at detecting the need for intensive care, with only $51 \%$ of patients requiring admission to intensive care units having CURB-65-defined severe disease (a score of $3+$ ). ${ }^{4}$ In addition, $20 \%$ of deaths occur in patients with CURB-65 scores of 2 or lower, and there was a mortality of $8 \%$ in the BTS audit for patients with a score of $2 .{ }^{4}$ Consequently, additional prognostic rules (eg the Infectious Diseases Society of America/ American Thoracic Society (IDSA/ATS) minor criteria and SMART-COP) have been designed, but as yet do not have the simplicity and proven validity of the CURB-65 score. Additional clinical indicators of severe disease, such as bilateral or multilobar consolidation, positive blood cultures, acidosis, hypoalbuminaemia and hypoxia, should be used to support risk stratification by CURB-65. ${ }^{8}$ Numerous biomarkers have also been tested for their utility in improving risk stratification of patients with CAP, including C-reactive protein (CRP), pro-calcitonin (PCT), cytokines and several stress hormones. ${ }^{9}$ Of these, CRP is the most readily available and does provide additional prognostic data. A CRP of $<100 \mathrm{mg} / \mathrm{l}$ is independently associated with a lower mortality and $>100 \mathrm{mg} / \mathrm{l}$ with increased risk of complication by CPE (Table 3). ${ }^{10,11}$

\section{Treatment}

Patients admitted to hospital are treated with a combination of a $ß$ lactam and a macrolide, whereas patients treated as outpatients are given single-agent therapy. ${ }^{8}$ The rationale for dual therapy in patients who are admitted is to cover CAP caused by atypical organisms (ie M. pneumoniae, C. pneumophila and L. pneumophila) as well as by S. pneumoniae. Some (but not all) retrospective data show that dual therapy is associated with significantly lower mortality compared with single-agent $B$ lactam (eg 2.9\% versus $11.4 \%$ for patients with a CURB-65 score of 2, and $11.1 \%$ versus 19.8 for a score of $3+) .{ }^{12}$ The size of the effect is unlikely to be purely related to treatment of atypical organisms, 
Table 3. Potential value of CRP as a marker of degree of inflammation for patients presenting with CAP.

\section{Potential role}

Distinguishing bacterial vs viral causes of CAP

Risk stratification

Duration of antibiotics

\section{CRP level and use}

$>100 \mathrm{mg} / \mathrm{l}$ suggests bacterial infection

$<100 \mathrm{mg} / \mathrm{l}$ indicates lower mortality

$>250 \mathrm{mg} / \mathrm{l}$ indicates increased mortality by $15 \%$ for patients with a CURB-65 score of $3+$

Identification of complicated CAP

$>100 \mathrm{mg} / \mathrm{l}$ indicates increased risk for CPE

Failure to fall by $50 \%$ at $96 \mathrm{~h}$ compared with admission increased risk of mortality (OR 24) and complications (OR 15)

No data yet; similar use of PCT reduced duration of antibiotics from 12 to 5 days

\section{Notes}

Only partially helpful? (PCT might be better)

Ref. 10

Ref. 10

Ref. 11

Ref. 10

Ref. 17

$\mathrm{CAP}=$ community-acquired pneumonia; $\mathrm{CPE}=$ complicated parapneumonic effusions; $\mathrm{OR}=$ odds ratio.

which in total cause only 20-25\% of CAP cases. Macrolides have significant anti-inflammatory effects, which has been exploited for their long-term use in bronchiectasis and cystic fibrosis, and might be beneficial in CAP. Inflammation is required to control microbial numbers, but it causes consolidation and, therefore, the hypoxia associated with CAP. It also contributes to the development of acute respiratory distress syndrome (ARDS) and septic shock, both of which have a high mortality. Hence, it is possible that dual therapy with a macrolide in addition to a $\beta$ lactam might improve mortality through modulation of the inflammatory response; if so, switching to a single-agent $\beta$ lactam in patients with proven S. pneumoniae CAP might not be beneficial. The potential detrimental effects of excess inflammation might be why statin therapy is associated with improved outcomes in $\mathrm{CAP}^{13}$ and has stimulated trials of systemic corticosteroids in patients with CAP. A recent randomised controlled trial supported the potential therapeutic benefit of systemic corticosteroids for patients with CAP; those given dexamethasone (5 mg intravenously on four occasions) had a more rapid fall in CRP, thus demonstrating a significant effect on the inflammatory response, and a shorter hospital stay (6.5 versus 7.5 days) compared with controls, with no difference in other outcomes. ${ }^{14}$ However, more research is required on the risks, potential benefits and optimum agents before anti-inflammatory therapy becomes routine for patients with CAP.

There are several problem areas with antibiotic treatment for CAP, including the best choice of antibiotic for patients who are allergic to penicillin. For mild or moderate disease, single-agent macrolide is acceptable but is probably not adequate for severe disease because of the risk of undertreating S. pneumoniae and $S$. aureus infection. For patients with a non-anaphylactic penicillin allergy, a second- or third-generation cephalosporin could be used. However, for patients with severe penicillin allergy, all related antibiotics should be avoided, including cephalosporins and penems, and limiting the alternatives to vancomycin, teicoplanin, linezolid or moxifloxacin. Another problem is overtreatment. Only 5\% of S. pneumoniae isolates in the UK are penicillin resistant and, because this is the result of changes to penicillinbinding proteins, most cases are only partially resistant. ${ }^{15}$
Therefore, amoxicillin is adequate therapy for most patients with CAP that is not caused by atypical microorganisms. Only the small proportion of patients with CAP resulting from $S$. aureus or Gram-negative bacteria require extended spectrum $B$ lactams, such as co-amoxiclav or cefuroxime, and these agents should be reserved for patients with a CURB-65 score of $3+$. Strict adherence to these guidelines reduced the use of cephalosporins for patients with CAP by $70 \%$ without affecting outcomes, ${ }^{16}$ which should help reduce Clostridium difficile infections as well as treatment costs.

Another area of potential overtreatment is the duration of antibiotic therapy, which is traditionally 7 days for patients admitted to hospital, but increasing to 14 days for patients with severe CAP or infected with $S$. aureus, atypical organisms or Gram-negative bacteria. A shorter duration of antibiotic treatment might be adequate for many patients with CAP, but inadequate for others, contributing to an increased risk of complications. An important randomised controlled trial used the levels of the serum marker of inflammation PCT to identify when patients can stop antibiotics. ${ }^{17}$ The physicians aimed to stop antibiotics when the PCT level fell to $<0.25 \mu \mathrm{g} / \mathrm{l}$; this reduced the median duration of antibiotic use from 12 to five days, with no differences in adverse outcomes between the two groups. These data suggest a more intelligent approach to duration of antibiotic therapy but should be replicated, preferably using a more readily available inflammatory marker, such as CRP.

There is a range of possibilities to be considered if a patient with CAP fails to improve (Table 4). The most common infective complication directly related to CAP is CPE, occurring in approximately $7 \%$ of patients admitted to hospital. Local pleural inflammation associated with the underlying consolidation is thought to cause the small parapneumonic effusions that are common in patients with CAP. Parapneumonic effusions become a CPE if there is evidence of infection of the pleural space, either because the pleural fluid contains detectable bacteria, has a low $\mathrm{pH}(<7.2)$, or is visibly turbid because of neutrophilia, or because imaging demonstrates that loculations have formed between the visceral and parietal pleura. 
Table 4. Reasons for failure to improve for patients found to have CAP.

\begin{tabular}{|c|c|c|}
\hline Category & Examples & Notes \\
\hline \multirow[t]{5}{*}{ Inadequate treatment } & Dose too low and/or wrong route & eg malabsorbing (rare) \\
\hline & $\begin{array}{l}\text { Atypical organism and not given macrolide } \\
\text { treatment }\end{array}$ & Uncommon; two weeks treatment recommended \\
\hline & Antibiotic-resistant Streptococcus pneumoniae & Suspect if recent foreign travel \\
\hline & $\begin{array}{l}\text { Unusual organism; eg MRSA or Pseudomonas } \\
\text { aeruginosa }\end{array}$ & Rare, $<1 \%$ cases of CAP; no response to empirical antibiotics \\
\hline & Bronchial obstruction & eg due to lung cancer \\
\hline \multirow[t]{2}{*}{ Non-infective complications } & ARDS & Usually early in admission \\
\hline & Septic shock & Usually early in admission \\
\hline \multirow[t]{4}{*}{ Infective complications } & CPE and/or empyema & $7 \%$ of cases, increasing incidence \\
\hline & Lung abscess, pericarditis, metastatic spread & All rare \\
\hline & Intravenous catheter site infection & Consider MRSA \\
\hline & Clostridium difficile diarrhoea & Associated with co-amoxiclav and/or cephalosporins \\
\hline \multirow[t]{3}{*}{ Not CAP: another lung infection } & Subacute lung infection & eg mycobacteria, Nocardia, Aspergillus and lung abscess \\
\hline & Pneumocystis jirovecii pneumonia & Can be a diagnostic problem if not known to be HIV positive \\
\hline & Metastatic infection & eg Staphylococcus aureus, Lemierre's syndrome \\
\hline \multirow{5}{*}{$\begin{array}{l}\text { Not CAP: non-infectious lung } \\
\text { disease }\end{array}$} & Pulmonary embolus and/or lung cancer & Both can cause consolidation plus some inflammation \\
\hline & Pulmonary oedema & Usually little inflammation \\
\hline & ARDS & eg associated with septicaemia \\
\hline & Alveolar cell carcinoma and/or lymphoma & Dense consolidation, usually little inflammation \\
\hline & $\begin{array}{l}\text { Pulmonary eosinophilia, organising pneumonia, } \\
\text { hypersensitivity pneumonitis, vasculitis and ABPA }\end{array}$ & $\begin{array}{l}\text { All cause lung shadowing with a significant inflammatory } \\
\text { response }\end{array}$ \\
\hline
\end{tabular}

ABPA = allergic bronchopulmonary aspergillosis; ARDS = acute respiratory distress syndrome; CAP = community-acquired pneumonia; MRSA = meticillin-resistant Staphylococcus aureas.

Loculations are best detected by pleural ultrasound, and ultrasound should also be used to optimise placement of the pleural drains that most patients with CPE require to ensure control of infection and minimise long-term loss of lung function resulting from pleural thickening. Compared with uncomplicated CAP, patients with a CPE have a longer hospital admission (mean of 15 versus 7 days) and duration of antibiotic treatment (3-4 weeks), as well as a high rate of surgical intervention $(20-30 \%)$ and a significant mortality (30\% if over 65 years of age). ${ }^{10,11}$ Importantly, the incidence of CPE is increasing worldwide, ${ }^{3}$ stressing the need for a better understanding of the pathogenesis of pleural infection and more effective management strategies.

\section{Mortality}

In a recent UK-wide BTS audit, the 30-day mortality of patients with CAP was $18 \%,{ }^{4}$ substantially higher than the $5 \%$ and $10 \%$ identified by previous studies. This high mortality might reflect the high proportion of older patients in the UK audit, with two thirds above 65 years of age compared with only half for similar data on patients with CAP from Germany. ${ }^{18}$ However patients over 65 years of age in the German study had a mortality rate of $10 \%$, nearly half that for all ages in the UK audit. The worryingly high mortality for patients with CAP in the UK needs further investigation and the potential reasons why explored in detail. CAP is also associated with a significant late mortality, perhaps related to cardiovascular events, with an additional $6 \%$ of patients aged over 65 years dying between 30 and 180 days after diagnosis. ${ }^{18}$

\section{Prevention}

The relatively high mortality of CAP demonstrates that prevention is important. Behavioural factors that predispose to CAP include smoking (estimated to be responsible for over $30 \%$ of cases of CAP $)^{19}$ and alcohol abuse, both of which can be modified to reduce the incidence of CAP. Owing to the close association of pneumonia with preceding respiratory virus infection, vaccination against influenza is also highly effective at preventing CAP. By contrast, the existing adult S. pneumoniae vaccine (Pneumovax ${ }^{\circledR}$ ) does not prevent pneumonia and the rationale for its use in older people is to prevent sepsis rather than lung infection. ${ }^{20}$ The new conjugated vaccine might be more immunogenic than Pneumovax, but is routinely used only for children. As yet there are no data on whether vaccination of adults with the conjugated vaccine prevents CAP; trials are in progress and could alter vaccination policy in the future. 


\section{Conclusions}

Despite the ready availability of antibiotics and vaccines for important respiratory pathogens, CAP remains a significant and increasingly common medical problem in the industrialised world, with a substantial rate of complication and mortality. In the future, routine use of biomarkers to improve risk stratification and tailor management to individual patients and possibly modulation of CAP-associated inflammation might result in improved outcomes. As well as clinical trials of different management and preventative treatments, further basic research is required on host-microbial interactions in the lung so that clinicians can fully understand why CAP develops and so enable the design of novel therapeutic strategies.

\section{References}

1 Trotter CL, Stuart JM, George R et al. Increasing hospital admissions for pneumonia, England. Emerg Infect Dis 2008;14:727-33.

2 Woodhead M, Welch CA, Harrison DA et al. Community-acquired pneumonia on the intensive care unit: secondary analysis of 17,869 cases in the ICNARC Case Mix Programme Database. Crit Care 2006;10:S1.

3 Grijalva CG, Zhu Y, Nuorti JP et al. Emergence of parapneumonic empyema in the USA. Thorax 2011;66:663-8.

4 Lim WS, Woodhead M, British Thoracic Society. British Thoracic Society adult community acquired pneumonia audit 2009/10. Thorax 2011;66:548-9.

5 Lim WS, Macfarlane JT, Boswell TC et al. Study of community acquired pneumonia aetiology (SCAPA) in adults admitted to hospital: implications for management guidelines. Thorax 2001;56:296-301.

6 Boyd AR, Orihuela CJ. Dysregulated inflammation as a risk factor for pneumonia in the elderly. Aging Dis 2011;2:487-500.

7 Baxendale HE, Brown JS. Mechanisms of immune protection to pneumococcal infection in the young and the elderly. In: Thiel A (ed), Immunosenescence. Basel: Springer Basel AG, 2012:85-126.

8 Lim WS, Baudouin SV, George RC et al. BTS guidelines for the management of community acquired pneumonia in adults: update 2009. Thorax 2009;64:iii1-55.
9 Brown JS. Biomarkers and community-acquired pneumonia. Thorax 2009;64:556-8.

10 Chalmers JD, Singanayagam A, Hill AT. C-reactive protein is an independent predictor of severity in community-acquired pneumonia. Am J Med 2008;121:219-25.

11 Chalmers JD, Singanayagam A, Murray MP et al. Risk factors for complicated parapneumonic effusion and empyema on presentation to hospital with community-acquired pneumonia. Thorax 2009;64:592-7.

12 Tessmer A, Welte T, Martus P et al. Impact of intravenous $\beta$-lactam/ macrolide versus $\beta$-lactam monotherapy on mortality in hospitalized patients with community-acquired pneumonia. J Antimicrob Chemother 2009;63:1025-33.

13 Chalmers JD, Singanayagam A, Murray MP et al. Prior statin use is associated with improved outcomes in community-acquired pneumonia. Am J Med 2008;121:1002-7.

14 Meijvis SC, Hardeman H, Remmelts HH et al. Dexamethasone and length of hospital stay in patients with community-acquired pneumonia: a randomised, double-blind, placebo-controlled trial. Lancet 2011;377:2023-30.

15 Blackburn RM, Henderson KL, Lillie $\mathrm{M}$ et al. Empirical treatment of influenza-associated pneumonia in primary care: a descriptive study of the antimicrobial susceptibility of lower respiratory tract bacteria (England, Wales and Northern Ireland, January 2007-March 2010). Thorax 2011;66:389-95.

16 Chalmers JD, Singanayagam A, Akram AR et al. Safety and efficacy of CURB65-guided antibiotic therapy in community-acquired pneumonia. J Antimicrob Chemother 2011;66:416-23.

17 Christ-Crain M, Stolz D, Bingisser R et al. Procalcitonin guidance of antibiotic therapy in community-acquired pneumonia: a randomized trial. Am J Respir Crit Care Med 2006;174:84-93.

18 Klapdor B, Ewig S, Pletz MW et al. Community-acquired pneumonia in younger patients is an entity on its own. Eur Respir J 2012;39:1156-61.

19 Almirall J, Gonzalez CA, Balanzo X et al. Proportion of communityacquired pneumonia cases attributable to tobacco smoking. Chest 1999;116:375-9.

20 Moberley SA, Holden J, Tatham DP et al. Vaccines for preventing pneumococcal infection in adults. Cochrane Database Syst Rev 2008;23:CD000422.

Address for correspondence: Dr JS Brown, University College Hosptial, Respiratory Medicine, 235 Euston Road, London NW1 2BU.

Email: jeremy.brown@ucl.ac.uk 